Article

\title{
Synergistic Effect of Expanded Graphite-Silane Functionalized Silica as a Hybrid Additive in Improving the Thermal Conductivity of Cementitious Grouts with Controllable Water Uptake
}

\author{
Ilayda Berktas ${ }^{1}{ }^{\circ}$, Ali Nejad Ghafar ${ }^{2}$, Patrick Fontana ${ }^{2}$, Ayten Caputcu ${ }^{3}$, \\ Yusuf Menceloglu 1,4 (D) and Burcu Saner Okan 1,* \\ 1 Sabanci University Integrated Manufacturing Technologies Research and Application Center \& Composite \\ Technologies Center of Excellence, Teknopark Istanbul, Pendik, Istanbul 34906, Turkey; \\ berktas@sabanciuniv.edu (I.B.); yusufm@sabanciuniv.edu (Y.M.) \\ 2 Department Infrastructure and Concrete Construction, RISE Research Institutes of Sweden, 11428 Stockholm, \\ Sweden; ali.nejad.ghafar@ri.se (A.N.G.); patrick.fontana@ri.se (P.F.) \\ 3 Cimsa Cimento Sanayi A. S., Toroslar Mah. Tekke Cad., Yenitaskent, \\ Mersin 33013, Turkey; A.CAPUTCU@cimsa.com.tr \\ 4 Faculty of Engineering and Natural Sciences, Sabanci University, Orhanli, Tuzla, Istanbul 34956, Turkey \\ * Correspondence: burcu.saner@sabanciuniv.edu
}

Received: 13 May 2020; Accepted: 11 June 2020; Published: 10 July 2020

\begin{abstract}
Recently, a growing demand for geothermal applications has led to the exploitation of energy efficiently by developing grouting materials in the borehole between pipes and the ground. Therefore, the current study developed newly formulated cementitious grouts by the integration of expanded graphite (EG)-based hybrid additives synthesized by building chemical bridges between silica particles and EG in the presence of amino functional silane coupling agents. These produced hybrid additives with controlled EG and silica ratios were utilized in grout mixtures used in borehole heat exchangers to enhance the thermal conductivity. According to the optimization study on the formulation development of grout mixtures with bentonite, silica sands, cement, and superplasticizer by adding neat EG and EG-based hybrids, the relationship between the carbon amount and water demand was found to have a significant impact on thermal conductivity. The highest thermal conductivity value of $2.656 \mathrm{~W} / \mathrm{mK}$ was achieved by the incorporation of $5 \mathrm{wt} \%$ hybrid additive with the ratio silica/EG of 1:5 compared to the reference grout, which showed a thermal conductivity of $2.373 \mathrm{~W} / \mathrm{mK}$. Therefore, the enhancement in thermal conductivity was dependent on the increase in the EG content and also the additive loading ratio, resulting in a slight increase in the water demand.
\end{abstract}

Keywords: expanded graphite; silanization; hybridization; thermal conductivity; cementitious grout; cement-based composites; ground source heat exchangers

\section{Introduction}

New research on renewable energy has been increasing due to the growing demand for energy all over the world. At this point, shallow geothermal energy systems have attracted enormous research interest owing to their advantages, such as reducing $\mathrm{CO}_{2}$ emission, being weather independent in contrast to other renewable energy technologies, and finally, their availability in most lands [1]. In such systems, the most prominent feature of a ground loop heat exchanger is the backfill grout, which allows heat exchange between the heat-carrier fluid in pipes and the surrounding formations [2]. Therefore, the thermal conductivity of the backfill grout should be as close as possible to the thermal 
conductivity of the surrounding formations to successfully exchange heat with minimum heat loss [3]. More specifically, as the thermal conductivity of the grout increases, more heat can be transferred through the borehole, and the length of the pipe to be constructed under the ground can be shortened, thus the installation costs are reduced [4].

According to the literature, several attempts have been carried out over the years to develop thermally enhanced grouts by using carbon nanotubes as nano-scaled additives. Lee et al. incorporated different concentrations of multi-walled carbon nanotubes (MWCNTs) into a grout consisting of cement, sand, and a surfactant, resulting in an improvement of the electrical resistance of $1.0 \%$ MWCNT-based grout with the filling rates of $100 \%, 75 \%, 50 \%$, and $25 \%$ as $0.449,0.575,0.846$, and $0.934 \mathrm{k} \Omega$, respectively [5]. In another study, Zhang and Li produced a cement-based composite for a thermally conductive layer in a deicing road system by integrating $3 \mathrm{wt} \%$ MWCNT and obtained a thermal conductivity of $2.83 \mathrm{~W} / \mathrm{mK}$ [6]. However, there are still challenges in the utilization of CNT in grouting mixtures, especially in mass production, due to their rigid surface, the requirement of an additional surface treatment, and high cost $[7,8]$. Therefore, the trend has so far been mainly focused on easily available materials to overcome the needs in backfill grouts used in borehole heat exchangers.

In previous studies carried out to improve the thermal conductivity of backfill grouts, various types of graphitic materials and treatment techniques have been tested to meet the defined requirements. For instance, Delaleux et al. studied the dispersion of different graphite loadings between 0 to $25 \mathrm{wt} \%$ with different intrinsic densities changing from 20 to $150 \mathrm{~kg} / \mathrm{m}^{3}$ in bentonite mortars, indicating an attainment of the highest thermal conductivity of $5 \mathrm{~W} / \mathrm{mK}$ by the addition of $5 \mathrm{wt} \%$ graphite with an internal density of about $100 \mathrm{~kg} / \mathrm{m}^{3}$ [9]. In another work, Lee et al. compared the effect of graphite and silica sand in the thermal conductivity of bentonite grout and stated that the use of $20 \%$ graphite increased the thermal conductivity of bentonite grout close to that in the geologic formation $(1.7-2.1 \mathrm{~W} / \mathrm{mK})$, whereas silica sand did not change the thermal conductivity even by the $60 \%$ loading [10]. Following this, Jobmann et al. investigated the influence of the temperature, water content, and density on thermal conductivity by using graphite as an admixture and achieved a thermal conductivity of $3 \mathrm{~W} / \mathrm{mK}$ with a water content of $14 \%$ and graphite content of $15 \%$ [11]. In addition to the thermal conductivity characteristic of grouts, it is possible to enhance the mechanical properties of cementitious grouts by polymer coating and the attachment of - $\mathrm{COOH}$ groups to graphite nanomaterials to improve their dispersion behaviors [12]. Therefore, the structural properties of graphitic materials should also be taken into consideration to allow improvement of the interfacial interactions with the cement matrix and the other components of the grout by tailoring the surface chemistry.

Among the graphitic materials, expanded graphite (EG) with a high carbon content has the benefit of improving the thermal conductivity of grout mixtures due to its remarkable thermal properties, low density, high porosity, planar geometry, and low price $[13,14]$. In one of the works, Bao et al. conducted infrared thermal image analysis to compare the performance of $20 \mathrm{wt} \%$ EGparaffin and $10 \mathrm{wt} \%$ graphene nanoplatelets (GNPs)-paraffin, which were used as the composite phase change materials in the developed cement-based composites, resulting in a better thermal regulatory performance with GNP due to its high thermal conductivity coefficient [15]. Furthermore, Zhang et al. fabricated EG/paraffin gypsum-based composite with $1 \mathrm{wt} \%$ carbon fiber and increased the thermal conductivity by $36.0 \%$ and $28.6 \%$ with the addition of $10 \%$ and $20 \%$ EG, respectively [16]. Accordingly, the recent investigations clearly show the potential of EG for use as a phase change material and its direct use in grout mixes without any required surface modification, especially in paraffin systems.

Another important criterion in grout mixtures is obtaining a uniform dispersion of the used additives. The incorporation of carbon-based materials into the cement mixture increases the viscosity and decreases the fluidity. Therefore, surface treatments on carbon-based materials gain high importance to enhance their dispersion in the cementitious matrix by providing efficient heat transfer. Luping et al. enhanced the interactions between graphene oxide (GO) and calcium silicate hydrate in a cement matrix by applying silane functionalization to form covalent bonds and provide better 
dispersion [17]. Moreover, Wang et al. improved the fluidity and rheological properties of cement paste and prevented the aggregation of particles by applying copolymerization on silane-modified GO [18]. These studies stated that the working performance of cement mixtures can be raised by the silanization of the selected additive. Silica has also gained considerable attention due to its ease of distribution, high modulus, electrical insulation, hardness, and extreme temperature sustainability [19]. Furthermore, the incorporation of silica into the cementitious mixture/mortar can improve the interfacial bonding of cement hydrates with modified carbon-based materials during the hydration reaction and a more compact structure can be obtained by filling the remaining voids in hydrated cement paste [12,20-22]. Especially, the development of silica-based hybrid additives by using graphitic structures can noticeably influence the performance of grout used in shallow geothermal energy systems. Numerous attempts have focused on the attachment of silica particles on the surface of graphite and graphene-based structures to achieve a high thermal conductivity in the field of improving the properties of the latex and electronic industry as high-performance thermal interface materials [23,24].

To the best of our knowledge, the performance of EG and silica in combined structures has not yet been sufficiently evaluated in grout mixes and there are very limited studies about the utilization of hybrid additives for the development of thermally enhanced grout composites. In order to maintain effective interfacial bonding between EG and the cementitious grout composites, the dispersibility of the graphitic materials added to the grout mix should be enhanced by surface treatment [25]. To overcome the low dispersibility of EG in cementitious grout composite, a new approach has been carried out by modifying the surface of EG with silane coupling agents in order to increase the surface hydrophilicity and interfacial interactions between EG and the cement matrix. Surface functionalization by organo-silanes is one of the efficient ways to improve the chemical adhesion between two materials [26]. Several studies have been conducted to improve the rheological properties and/or strength of cement mortars by the addition of carbon-based materials [27].

In the present study, the silanization process was employed to develop new forms of EG-based hybrid additives by changing the amounts of functionalized silica and EG for the fabrication of thermally enhanced grout composites to enhance the interfacial interactions between the developed additives, bentonite, and the other grout components. In order to understand the extent of the influence of the carbon content on the performance of the developed cementitious grout composites, chemical compositions of hybrid additives were tailored by changing the silica and EG contents. Detailed spectroscopic and macroscopic studies were carried out to confirm the structural formation of EG-based hybrid additives. Then, the performances of the produced EG-based hybrid additives were tested in the grout mixes formulated to obtain the highest possible thermal conductivity with the proper rheological properties.

\section{Materials and Methods}

\subsection{Materials}

In this investigation, 3-aminopropyl triethoxysilane (APTES $>98 \%$ ), acetic acid, and tetrahydrofuran (THF) were obtained from Sigma-Aldrich, USA. Amorphous silica $\left(\mathrm{SiO}_{2}\right)$ was obtained from Merck, Germany. Expanded graphite-GFG5 with a $5-\mu \mathrm{m}$ particle size was obtained from SGL Carbon, Germany. Portland cement CEM I 42.5 R from Cimsa, Turkey, silica sand (AFS 30-35 and AFS 60-70) from Kumsan Co, Turkey, superplasticizer (SMRC-310S) from Sika, Turkey and bentonite from Canbensan Bentonite, Turkey were used in the preparation of the grout composites. A Malvern 3000 laser diffraction particle size analyzer was used to analyze the particle size distribution of the constituents. Table 1 summarizes the particle size distributions of cement, bentonite, silica sands AFS 30-35, and AFS 60-70 regarding D10, D50, and D90, which are the intercepts for $10 \%, 50 \%$, and $90 \%$ of the cumulative mass of the analysis results. The product of silica sand, AFS 30-35 with D90 $=912 \mu \mathrm{m}$, has the largest particle size among the samples, whereas cement with D90 $=46.4 \mu \mathrm{m}$ has the smallest one. 
Table 1. Particle size distributions (D10, D50, and D90) of cement, bentonite, and two types of silica sands according to $10 \%, 50 \%$, and $90 \%$.

\begin{tabular}{cccc}
\hline Constituent Type & D10 $(\mu \mathrm{m})$ & D50 $(\boldsymbol{\mu m})$ & D90 $(\boldsymbol{\mu m})$ \\
\hline Cement & 3.72 & 17.8 & 46.4 \\
\hline Bentonite & 9.21 & 40.6 & 131 \\
\hline Silica Sand AFS 30-35 & 401 & 621 & 912 \\
\hline Silica Sand AFS 60-70 & 134 & 250 & 454 \\
\hline
\end{tabular}

\subsection{Synthesis of Functionalized Silica}

Silane coupling agents have considerable influence on the dispersion of fillers and the improvement of interfacial interactions between fillers and the constituents in a cement matrix. The dispersion quality of the selected fillers directly enhances the thermal, mechanical, and physical properties of nanocomposites since silane coupling agents act as bridge between silica and EG by increasing the compatibility with the component of grout mixes. APTES is one of the most commonly used silane coupling agents that connects chemically reactive functional amino groups to silica and hydrolysable groups on EG by the silanization process. In order to provide effective functionalization, the optimum APTES:silica ratio was determined as 2:1 by adjusting the weight to weight ratio of silica and silane amounts. In more detail, $1 \mathrm{~g}$ of silica was first mixed into $50 \mathrm{~mL}$ of distilled water. Then, $2 \mathrm{~mL}$ of APTES was dissolved into the mixture and the $\mathrm{pH}$ level of the solution was adjusted to 5.5 by adding acetic acid. Afterwards, the mixture was stirred at $80^{\circ} \mathrm{C}$ for $24 \mathrm{~h}$ to ensure completion of the silanization reaction. At the end of the reaction, the mixture was filtered by washing with water and ethanol twice in order to remove the remaining silane coupling agent. The filtrate was then dried in an oven at $70{ }^{\circ} \mathrm{C}$ for $24 \mathrm{~h}$. The detailed production process of APTES-functionalized silica particles with an average diameter of $130 \mathrm{~nm}$ can be found in our previous publication [28].

\subsection{Hybridization of EG with Functionalized Silica}

In this study, hybrid EG/silica additives modified by silane coupling agents were developed by tailoring surface functional groups. To produce a homogeneously dispersed EG solution, THF was selected as a reaction medium [29]. Accordingly, approximately $240 \mathrm{~g}$ of EG was added to $7.5 \mathrm{~L}$ of THF. The solution was then subjected to the ultrasonic dispersion process by a handheld ultrasonic homogenizer from Hielscher ultrasonics at room temperature for $30 \mathrm{~min}$. Afterwards, a previously prepared APTES-functionalized silica aqueous solution (Silica:APTES = 1:2, encoded as fSi) was slowly added into the EG-based suspension. The reaction was carried out through refluxing at $60^{\circ} \mathrm{C}$ overnight. After the reaction, the resultant material was obtained by centrifugation and then separated by decantation and dried at $70{ }^{\circ} \mathrm{C}$ overnight in a vacuum oven. While the silica to silane ratio in functionalized silica was kept as 1:2 in the hybrid additives, as summarized in Table 2, the ratios of functionalized silica and EG were adjusted as 1:1 and 1:5 encoded as H-EG-1 and H-EG-2 (also called as fSi/EG-based hybrid additive), respectively, to monitor the effect of the carbon content on the performance of the hybridized additives in the grout mixes.

Table 2. Summary of the synthesis conditions of EG-based hybrid additives.

\begin{tabular}{ccccc}
\hline Samples & Silica: APTES = 1:2(fSi) (g) & EG Amount (g) & Reaction Medium & Reaction Time (h) \\
\hline $\begin{array}{c}\text { H-EG-1 } \\
\text { (fSi:EG }=1: 1)\end{array}$ & 1 & 1 & THF & 24 \\
$\begin{array}{c}\text { H-EG-2 } \\
\text { (fSi:EG }=1: 5)\end{array}$ & 1 & 5 & THF & 24 \\
\hline
\end{tabular}




\subsection{Preparation of Grout Samples by Using Hybrid Additives}

Since the addition of EG-based materials affects the properties of neat grout composites, the optimization process is crucial to obtain the best possible properties. As shown in Table 3, the neat grout composition consisted of $65.69 \mathrm{wt} \%$ of silica sands, with different particle sizes named 30-35 AFS and 60-65 AFS; $33.94 \mathrm{wt} \%$ of cement; and $0.37 \mathrm{wt} \%$ of bentonite and superplasticizer. Then, EG-based hybrid additives were added with two different concentrations of $3 \mathrm{wt} \%$ and $5 \mathrm{wt} \%$ by tailoring the amount of bentonite and water uptake. The grout composites were mixed using a high-share dispersion system from VMA-Getzmann. While part of the prepared grout mix was separated each time for Marsh-cone and flow-table tests, the rest of the material was poured into disk-shaped molds ( $20 \mathrm{~mm}$ in height $\times 60 \mathrm{~mm}$ in diameter) and kept in a climate chamber under $100 \%$ relative humidity and $20^{\circ} \mathrm{C}$ for curing. The curing stages of the samples were examined on the 7th, 14th, and 28th days. Conductivity measurements were carried out on the mentioned days after the cement sample was poured into the mold and placed in a $100 \%$ moist environment at $20^{\circ} \mathrm{C}$. Since no significant change was observed on these days, thermal conductivity tests were carried out for the samples cured on the 7th day. The samples were then used to determine the thermal conductivity of the EG-based hybrid additive enhanced cementitious grout composites. Thermal conductivity tests were performed under $23{ }^{\circ} \mathrm{C}$ and $50 \% \pm 2 \%$ humidity. Further details about the mixing regime, sample preparation, and tests conducted for the thermal and rheological characterizations of the grout mixes can be found in the previous article [28].

Table 3. Reference grout formulation.

\begin{tabular}{cc}
\hline Component & wt $\%$ \\
\hline Cement & 33.94 \\
\hline Silica Sand (30-35 AFS) & 32.845 \\
\hline Silica Sand (60-65 AFS) & 32.845 \\
\hline Bentonite & 0.37 \\
\hline
\end{tabular}

\subsection{Characterization}

The morphologies of neat EG, silica and $\mathrm{fSi}: \mathrm{EG}=1: 1$ and $\mathrm{fSi}: \mathrm{EG}=1: 5$ hybrid additives were examined by scanning electron microscope (SEM, Leo supra 35VP field emission). The thermal properties of the prepared samples were assessed by a using thermal gravimetric analyzer over the temperature range of 25 to $1000{ }^{\circ} \mathrm{C}$ at a heating rate of $10^{\circ} \mathrm{C} / \mathrm{min}$ under the flow of nitrogen (TGA, Mettler Toledo TGA/DSC 3+). The crystallinity of the samples was analyzed by X-ray diffraction with a $\mathrm{CuK} \alpha$ radiation source (XRD, Bruker D2 PHASER Desktop). An X-ray photoelectron spectroscopy system (XPS, Thermo Scientific K-Alpha) was also used to examine the elemental composition of EG and its hybrid additives. The thermal conductivity of the silica/EG-based cementitious grout composites was investigated using a thermal conductivity analyzer (hot disk thermal constants analyser, TPS $2500 \mathrm{~S}$ ). The thermal conductivity tests were repeated with three different combinations of identical samples to obtain more accurate results and the reported values were the average of the three repetitions. The surface areas of the samples were measured by the Brunauer-Emmett-Teller technique using Micromeritics 3Flex surface analyzer equipment. The structural changes in carbon-based materials were characterized by Raman spectroscopy with a laser wavelength of $532 \mathrm{~nm}$ in the range of $100-3500 \mathrm{~cm}^{-1}$ (RAMAN, Renishaw inVia reflex raman microscopy system). 


\section{Results and Discussion}

\subsection{Structural Properties of Functionalized Silica and Silica-Modified Hybrid EG Additives}

Although the addition of EG as an additive improves the rheological, mechanical, and thermal properties of cementitious grout composites, a homogenous dispersion of EG is one of the main requirements to obtain a uniform composition. Therefore, the coverage of the EG surface with silanized silica might be an effective method to provide proper interaction between EG and other grout constituents. In the initial part of functionalization, silane coupling agents of APTES were attached covalently to the surface of the silica with their hydrolyzed groups in a water medium with adjusted $\mathrm{pH}$ as seen in Figure 1a. In other words, the formation of a network between three hydrolysable groups of silanes and $\mathrm{OH}$ groups of silica caused covalent interactions to preserve the uniformity in the structure. In the second part, amine functional groups were linked to the EG sheets and formed the hybrid additive as shown in Figure $1 b$.

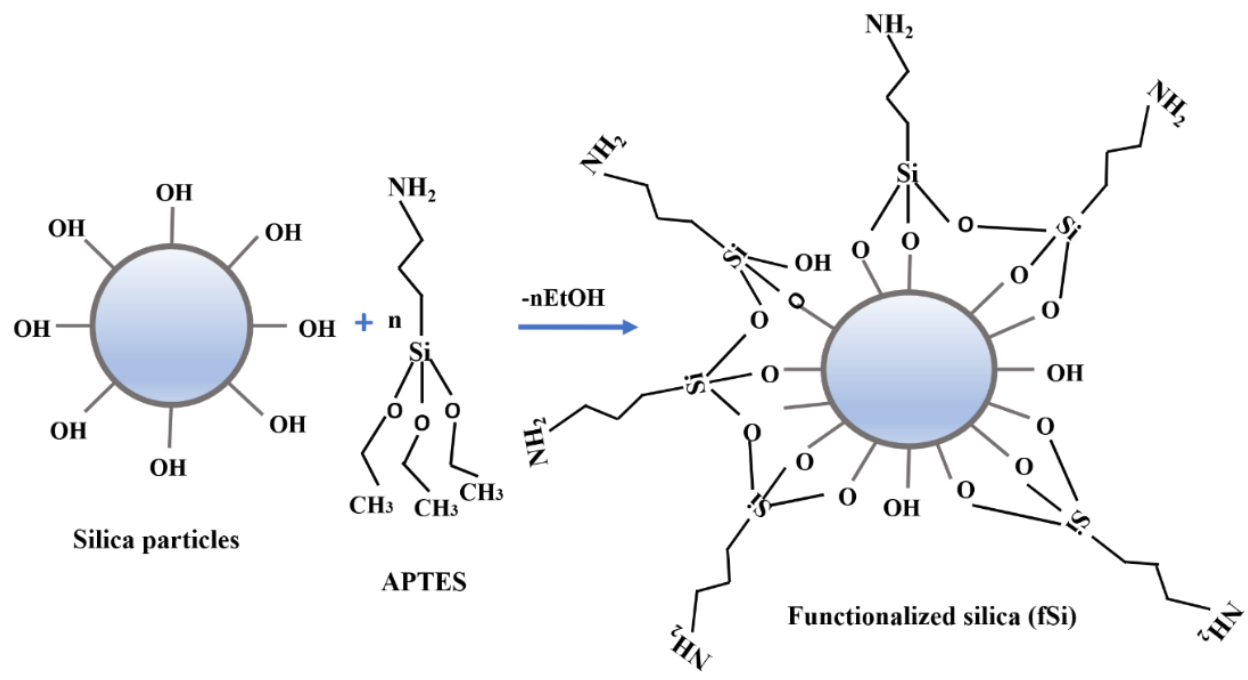

(a)

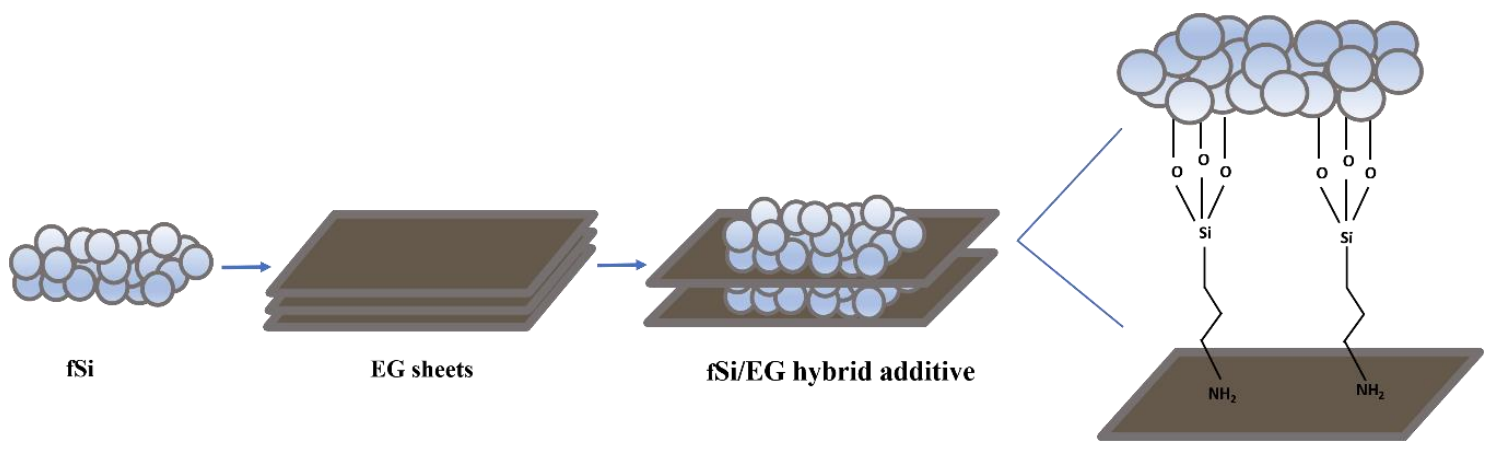

(b)

Figure 1. Schematic representation of (a) APTES-functionalized silica (fSi) and (b) fSi/EG-based hybrid additive.

In order to observe the influence of the $\mathrm{fSi} / \mathrm{EG}$ ratio on the thermal conductivity of the developed cementitious grouts, two different hybrid additives were produced and tested by changing the amounts of silica and EG as 1:1 and 1:5, while keeping the ratio of silica to APTES constant. The results showed that by changing the carbon content in the structure of the hybrid additive, the specific surface area of the hybrid noticeably changed. In the case of H-EG-1, the BET surface area was measured as $74 \mathrm{~m}^{2} / \mathrm{g}$ compared to that of neat EG, which was evaluated as $30 \mathrm{~m}^{2} / \mathrm{g}$. In the case of $\mathrm{H}-\mathrm{EG}-2$, as the carbon 
content in the hybrid structure was increased, the BET surface area was decreased down to $12 \mathrm{~m}^{2} / \mathrm{g}$. This is mainly due to the very high surface area of neat silica $\left(473.8 \mathrm{~m}^{2} / \mathrm{g}\right)$ compared to that of EG $\left(30 \mathrm{~m}^{2} / \mathrm{g}\right)$. Herein, as the carbon content was increased, the surface area of the hybrid was decreased.

Amino groups coming from APTES that are attached on the silica surface play a significant role in obtaining an ideal hybridization with the EG particles. Therefore, chemical composition analysis was carried out by XPS to monitor the changes in the chemical composition on the surface of the hybrid EG additives compared to neat silica and EG. Survey scans of EG, represented in Figure 2a, show two main peaks of C1s and O1s at 286 and $534 \mathrm{eV}$, respectively, indicating the presence of the carbon and oxygen content [30]. According to the XPS results tabulated in Table 4, EG contains $98 \%$ of carbon and $2 \%$ of oxygen in its structure, which makes it an excellent candidate for the enhancement of higher thermal conductivity than other carbon-based materials [31]. As seen in the XPS scans in Figure 2a and Table 4 , while neat silica consists of $60 \%$ oxygen, $3.3 \%$ carbon, and $36.7 \%$ silicon, there is a decrease in the oxygen and silicon contents, and an increase in the carbon and nitrogen contents in Si:APTES = 1:2, indicating successful silanization. Furthermore, after functionalization of the silica surface with APTES (fSi), nitrogen appeared at around $399 \mathrm{eV}$ in the survey scan of Si:APTES $=1: 2$. This is clear evidence of bonding between silica and APTES. Considering the scans of the hybrid additives, the increase in the carbon content and decrease in the nitrogen content clarify the formation of the hybrid structures. Herein, the increased carbon content is an indication of modification of the APTES-functionalized silica with EG. The results also showed that H-EG-2 had a higher carbon content and lower Si content compared to H-EG-1. This shows that it is feasible to control the surface composition using a proper chemical approach. Moreover, the deconvolution of the C1s, O1s, and N1s spectra for EG, silica, H-EG-1, and H-EG-2 were carried out to investigate the formation of the interfacial interaction between the components. In the $\mathrm{C} 1 \mathrm{~s}$ deconvolution shown in Figure $2 \mathrm{~b}$, the peaks at around $284.5 \mathrm{eV}$ belongs to $\mathrm{C}-\mathrm{C}$, indicating a highly ordered $\mathrm{sp}^{2}$ graphitic network [32,33]. Figure $2 \mathrm{c}$ shows the deconvolution of the O1s spectra of neat and hybrid structures, which showed peaks at around $532.8 \mathrm{eV}$, which are attributed to the presence of $\mathrm{O}-\mathrm{Si}$ in the structures [34]. Figure $2 \mathrm{~d}$ represents the deconvolution of the N1s spectra of functionalized silica and hybrid additives. The sharp N1s peak of functionalized silica at around $398.8 \mathrm{eV}$ indicates the presence of $\mathrm{NH}_{3}$ groups. After modifying the surface of EG with APTES-functionalized silica, the N1s peak became broader. Moreover, they were shifted to higher values and appeared at around 400 and $399.4 \mathrm{eV}$ for H-EG-1 and H-EG-2, respectively, indicating nitridation of the EG surface. In addition, there are alterations in the intensities of the N1s peaks of H-EG-2 (fSi:EG = 1:5) and H-EG-1 (fSi:EG = 1:1), coming from the formation of amine and charged amine moieties, respectively, in the hybrid structures [35].

Table 4. XPS results of silica, Silica: APTES =1:2, EG and its hybrid additives with different carbon contents.

\begin{tabular}{ccccc}
\hline Sample Name & Carbon (at\%) & Oxygen (at\%) & Silicon (at\%) & Nitrogen (at\%) \\
\hline Silica & 3.3 & 60 & 36.7 & - \\
\hline Silica: APTES = 1:2 & 26 & 43 & 27 & 3.1 \\
\hline EG & 98 & 2 & - & - \\
\hline H-EG-1 & 50.5 & 30.3 & 18.2 & 1 \\
\hline H-EG-2 & 88.2 & 6.7 & 3.2 & 1.7 \\
\hline
\end{tabular}




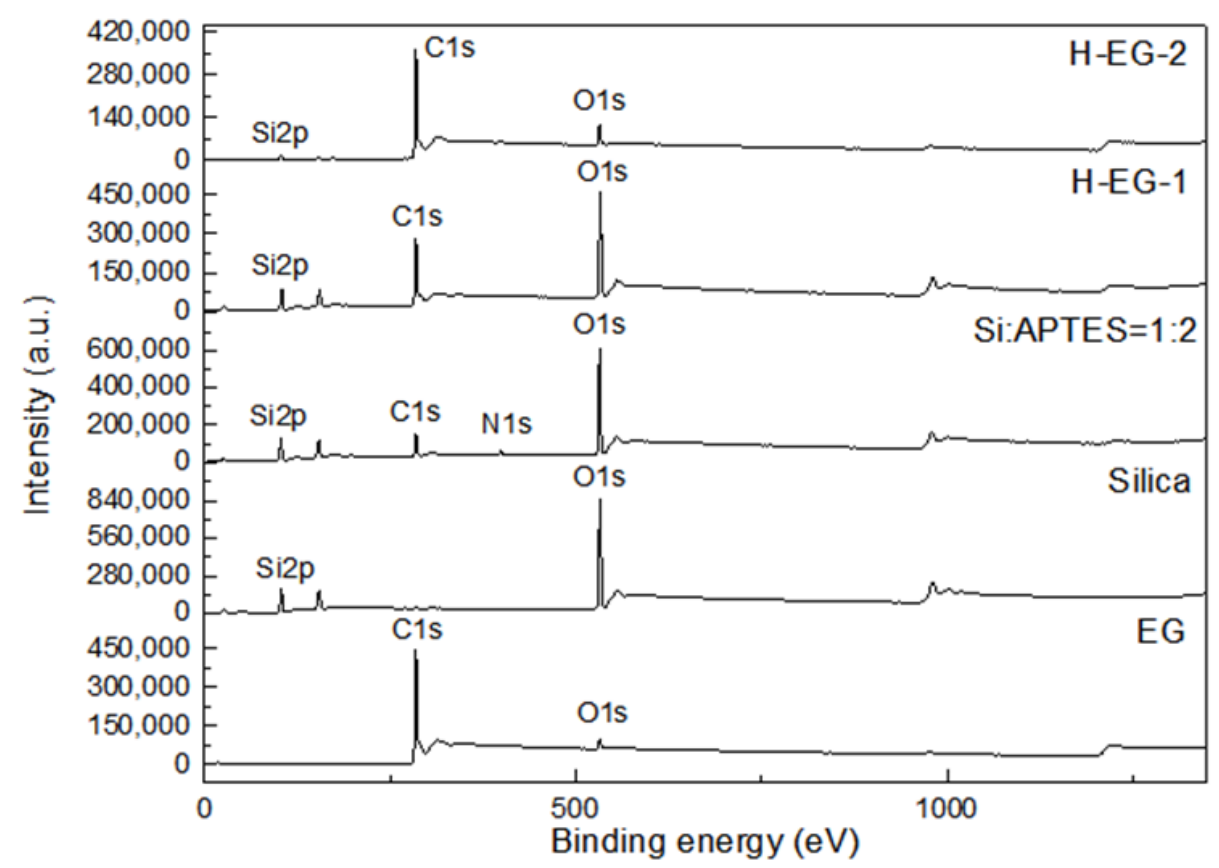

(a)

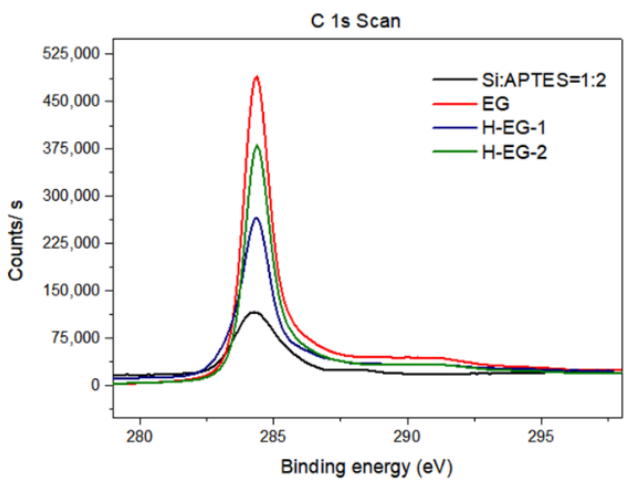

(b)

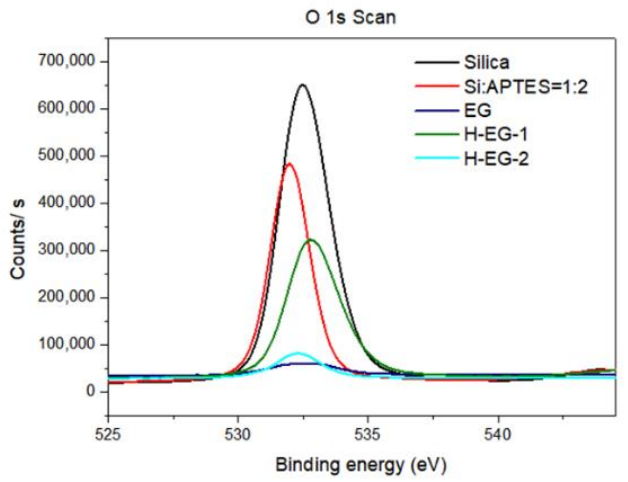

(c)

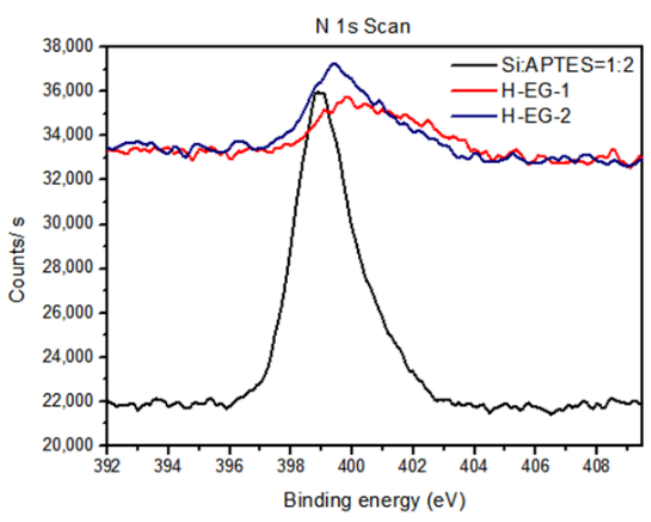

(d)

Figure 2. XPS characterization results: (a) XPS survey scan spectra, (b) C1s spectra, (c) O1s spectra, and (d) N1s spectra of silica, Silica:APTES = 1:2, EG, H-EG-1 and H-EG-2. 
XRD analysis was performed to monitor the changes in the crystallinity of the hybrid additives. Figure 3 exhibits the XRD spectra of silica, EG, H-EG-1, and H-EG-2. The broad XRD peak on the pattern of silica appeared at around $2 \theta=21^{\circ}$, belonging to (101) planes, revealing the amorphous structure of the silica [36], and the peak that appeared at around $2 \theta=43.6^{\circ}$ belongs to the metallic holder. EG shows a sharp peak at around $2 \theta=26.5^{\circ}$ belonging to the diffraction of (002) planes and a smoother peak at around $2 \theta=54.6^{\circ}$ belonging to the diffraction of (004) planes, indicating a crystalline structure [30]. As seen in Table 5, after the attachment of the silica particles on the EG surface, the crystallinity degree of EG decreases from $89 \%$ down to $70.6 \%$ and $68.7 \%$ in H-EG-1 and H-EG-2, respectively. This phenomenon can be observed in the XRD spectra, showing the decrease in the intensity of the (002) peak due to the amorphous structure of the silica, which affects the crystallinity of the hybrids.

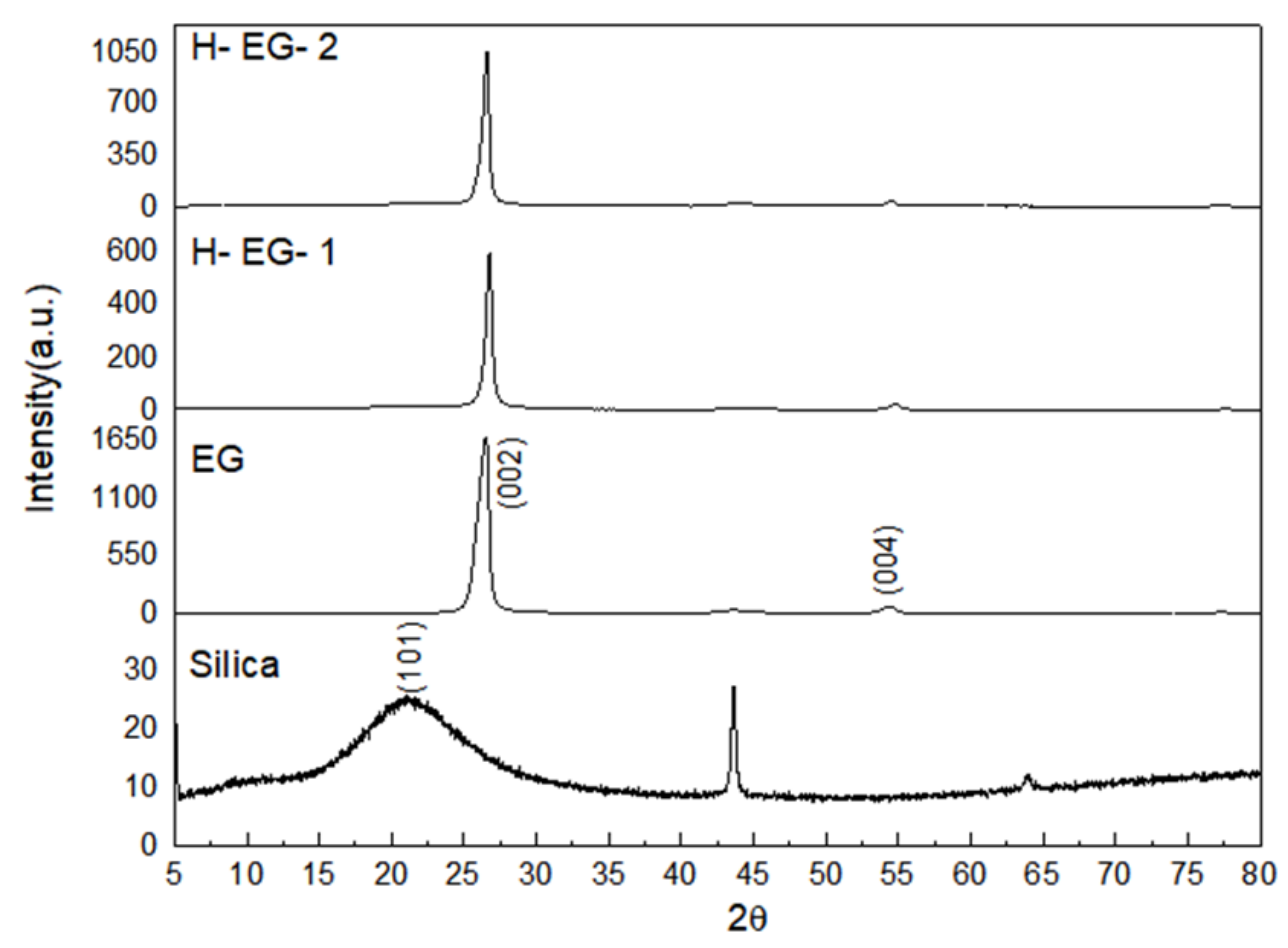

Figure 3. XRD spectra of silica, EG, H-EG-1, and H-EG-2.

Table 5. Crystallinity results from the XRD characterization.

\begin{tabular}{ccc}
\hline Sample Name & Crystallinity (\%) & Amorphous (\%) \\
\hline Silica & 58.7 & 41.3 \\
\hline Silica: APTES = 1:2 & 38.2 & 61.8 \\
\hline EG & 89 & 11 \\
\hline H-EG-1 & 70.6 & 29.4 \\
\hline H-EG-2 & 68.7 & 31.3 \\
\hline
\end{tabular}

Figure 4 exhibits the Raman spectra of silica, EG, H-EG-1, and H-EG-2. Three diffraction peaks appeared in the Raman spectrum of EG. The sharp and strong G-peak seen at around $1579 \mathrm{~cm}^{-1}$, the weak D-peak at $1350 \mathrm{~cm}^{-1}$, and the $2 \mathrm{D}$ band (the overtone of the $\mathrm{D}$ band) at $2700 \mathrm{~cm}^{-1}$ are indications of the graphitic carbon nature of EG. All three characteristic peaks known as D, G, and 2D, belonging to EG, are also seen in the Raman spectra of H-EG-1 and H-EG-2, with slight up-shifts compared to the peak positions of EG due to the impregnation of the silica particles and chemical doping on the surface of EG [37]. There is no Raman peak attributed to silica. As the carbon content 
of the hybrid additives increased (from H-EG-1 to H-EG-2), the intensity of the D peak decreased, indicating a reduction of the disorder in the lattice [38]. Furthermore, as shown in Table 6, the relative intensity $\left(\mathrm{I}_{\mathrm{D}} / \mathrm{I}_{\mathrm{G}}\right)$ of H-EG-2 was calculated as 0.52 , which is less than the $\mathrm{I}_{\mathrm{D}} / \mathrm{I}_{\mathrm{G}}$ ratios of H-EG-1 with 0.68 and neat EG with 0.61. This can be an indication of an increase in the order of the structure [32].

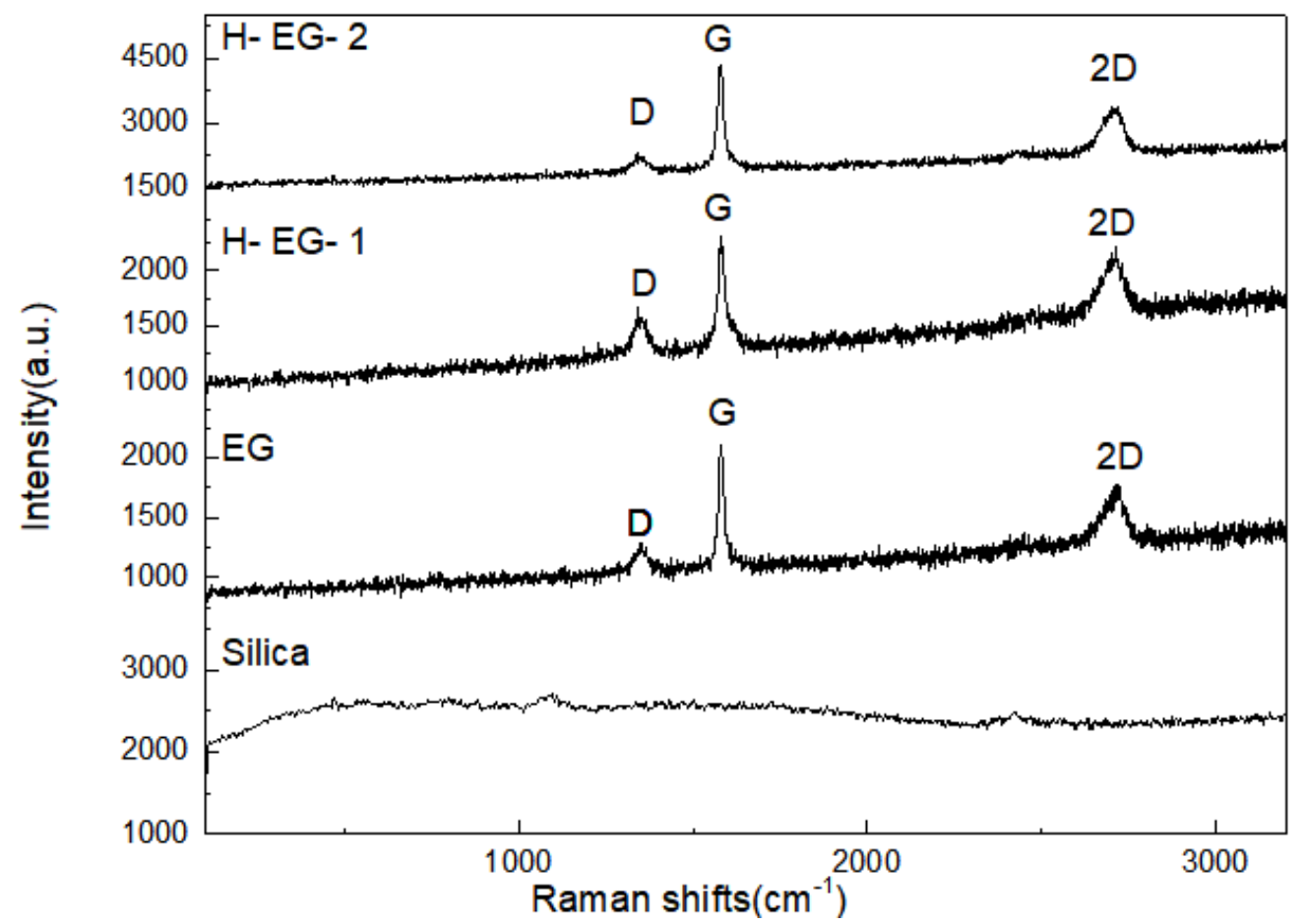

Figure 4. Raman spectra of silica, EG, H-EG-1, and H-EG-2.

Table 6. Raman peak intensities and $\mathrm{I}_{\mathrm{D}} / \mathrm{I}_{\mathrm{G}}$ ratios of EG, H-EG-1, and H-EG-2.

\begin{tabular}{ccccc}
\hline Sample Names & $\begin{array}{c}\text { D Peak Intensity } \\
\text { (a.u.) }\end{array}$ & $\begin{array}{c}\text { G Peak Intensity } \\
\text { (a.u.) }\end{array}$ & $\begin{array}{c}\text { 2D Peak Intensity } \\
\text { (a.u.) }\end{array}$ & $\mathbf{I}_{\mathbf{D} / \mathbf{I}_{\mathbf{G}}}$ \\
\hline EG & 1291.6 & 2108 & 1779 & 0.61 \\
\hline H-EG-1 & 1585.8 & 2309.4 & 2222.6 & 0.68 \\
\hline H-EG-2 & 2291.5 & 4364.1 & 3383.5 & 0.52 \\
\hline
\end{tabular}

\subsection{Thermal Degradation Behaviors of Neat and Silica-Modified EG Hybrid Additives}

TGA was conducted in order to investigate the thermal stability and evaluate the functional groups of the developed EG-based hybrid additives. Figure 5 shows the TGA curves of silica, Silica:APTES = 1:2, EG, H-EG-1, and H-EG-2. As seen in the TGA curves, there is an enormous decrease in the degradation profile with the functionalization of silica with APTES. After grafting the surface of EG with functionalized silica, the degradation profiles of both hybrid additives become more thermally stable compared to functionalized silica, showing successful bonding. The hybrid additives of H-EG-1 and H-EG-2 show an approximately $3 \%$ and $5 \%$ weight loss, respectively, at $1000{ }^{\circ} \mathrm{C}$. This alteration in weight loss comes from the differences in the carbon and silica ratio since H-EG-1 contains a high silica amount and thus shows better thermal stability than H-EG-2. 


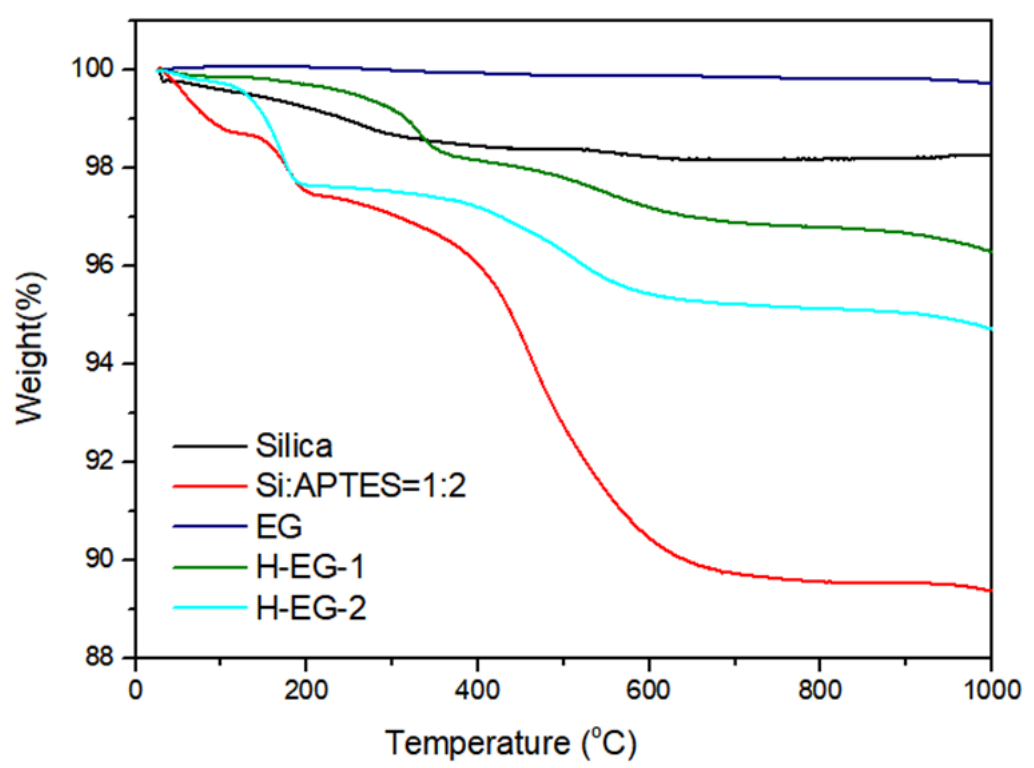

Figure 5. TGA curves of silica, Silica:APTES = 1:2, EG, H-EG-1, and H-EG-2.

\subsection{Morphological Properties of Neat and Hybrid Additives}

Figure 6 represents the SEM images of neat EG, neat silica, H-EG-1 (low carbon content), and H-EG-2 (high carbon content). The SEM image of neat EG in Figure 6a has sharp, rigid, and stacked layers. The SEM image in Figure $6 \mathrm{~b}$ shows the randomly distributed amorphous $\mathrm{SiO}_{2}$ particles. After the hybridization of EG with functionalized silica with the ratios of $\mathrm{fSi}: \mathrm{EG}=1: 1$ and $\mathrm{fSi}: \mathrm{EG}=1: 5$, as seen in in Figure $6 c, d$, both silica particles and rigid EG structures are observed clearly. Especially, the high moiety of the silica particles is seen in Figure $6 \mathrm{c}$ due to the presence of a high amount of fSi. To conclude, the morphological studies revealed that the silica particles were attached to the surface of EG with the help of silane-based coupling agents.

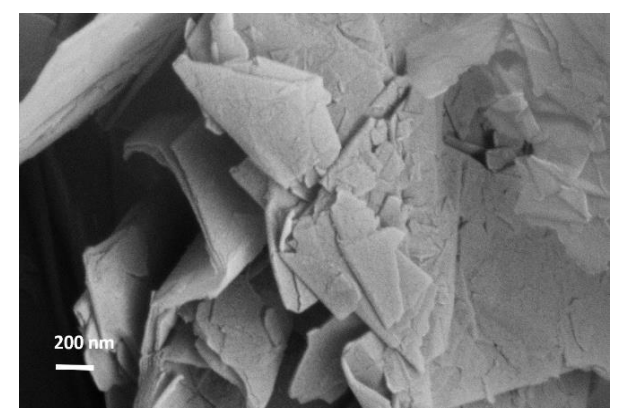

(a)

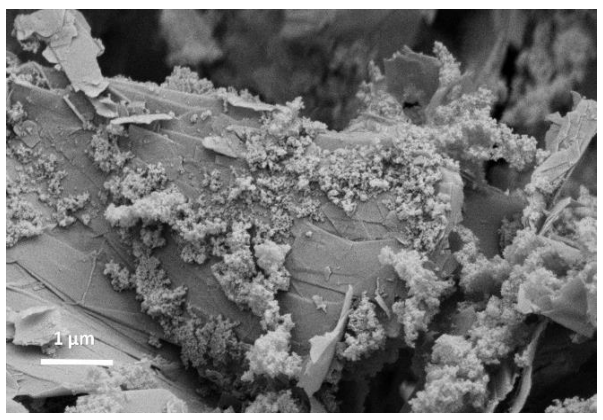

(c)

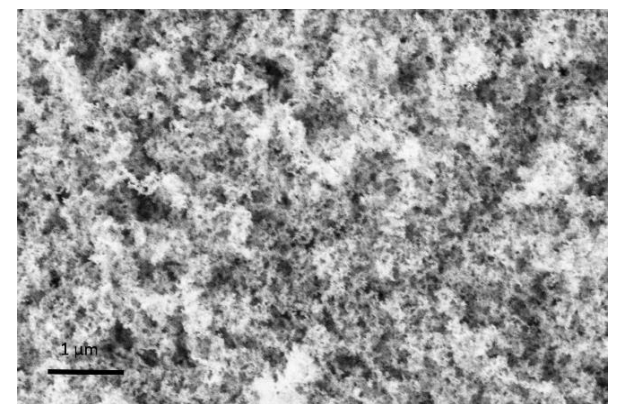

(b)

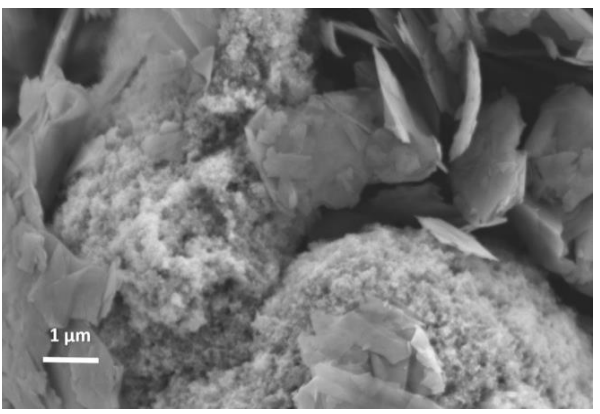

(d)

Figure 6. SEM images of: (a) neat EG (b) neat silica (c) fSi:EG = 1:1, and (d) fSi:EG = 1:5 hybrid additives. 


\subsection{Thermal Conductivity and Rheological Behaviors of Silica-EG Hybrid Additive Grout Composites}

The results of the thermal conductivity measurements of neat and grout samples with silica-EG hybrid additives with $3 \mathrm{wt} \%$ and $5 \mathrm{wt} \%$ loadings are presented in Table 7 . The flowability tests, bleeding tests, and density measurements of neat and grout samples conducted in fresh conditions are also given in the same table. The developed thermally enhanced grouts consist of cement, two silica sands with different particle size distribution curves, bentonite, superplasticizer, and two newly developed hybrid additives. The thermal conductivity of the cementitious grout produced with the same materials but with no additive (the reference grout) was measured as $2.373 \mathrm{~W} / \mathrm{mK}$ (test 1 ). The addition of $3 \mathrm{wt} \%$ of H-EG-1 into the reference grout mix increased the water demand from 650 to $750 \mathrm{~g}$ and as a result decreased the thermal conductivity from 2.373 to $2.175 \mathrm{~W} / \mathrm{mK}$. To examine the effect of the water content on the thermal conductivity, in test 3 , the amount of water was reduced from 750 to $730 \mathrm{~g}$, while the contents of the other components were constant. The result of the thermal conductivity measurement showed a slightly higher value $(2.429 \mathrm{~W} / \mathrm{mK})$ compared to the result of test $2(2.175 \mathrm{~W} / \mathrm{mK})$. In the comparison of test 2 and test 3 , as the water content was decreased by $2.66 \%$, the result of the thermal conductivity showed an increase of $9.34 \%$. This implies that in the cementitious grout composite, increasing the water content decreases the thermal conductivity. To solve this issue and improve the thermal conductivity effectively, the fSi/EG ratio in the hybrid additive structure was changed from 1:1 in H-EG-1 to 1:5 in H-EG-2, respectively, with the aim of increasing the carbon content. In addition, in test 4 , the content of the hybrid additive in the grout composition was increased from $3 \mathrm{wt} \%$ to $5 \mathrm{wt} \%$. Even though the result of test 4 showed a slightly higher water demand, the resulting thermal conductivity of the reformulated grout increased to $2.656 \mathrm{~W} / \mathrm{mK}$, with the rheological properties in the accepted ranges. This improvement is attributed to the low water demand but the high EG content in the grout composition produced with H-EG-2.

Table 7. Thermal conductivity results of reference grout and with the addition of H-EG-1 and H-EG-2 in different loading percentages.

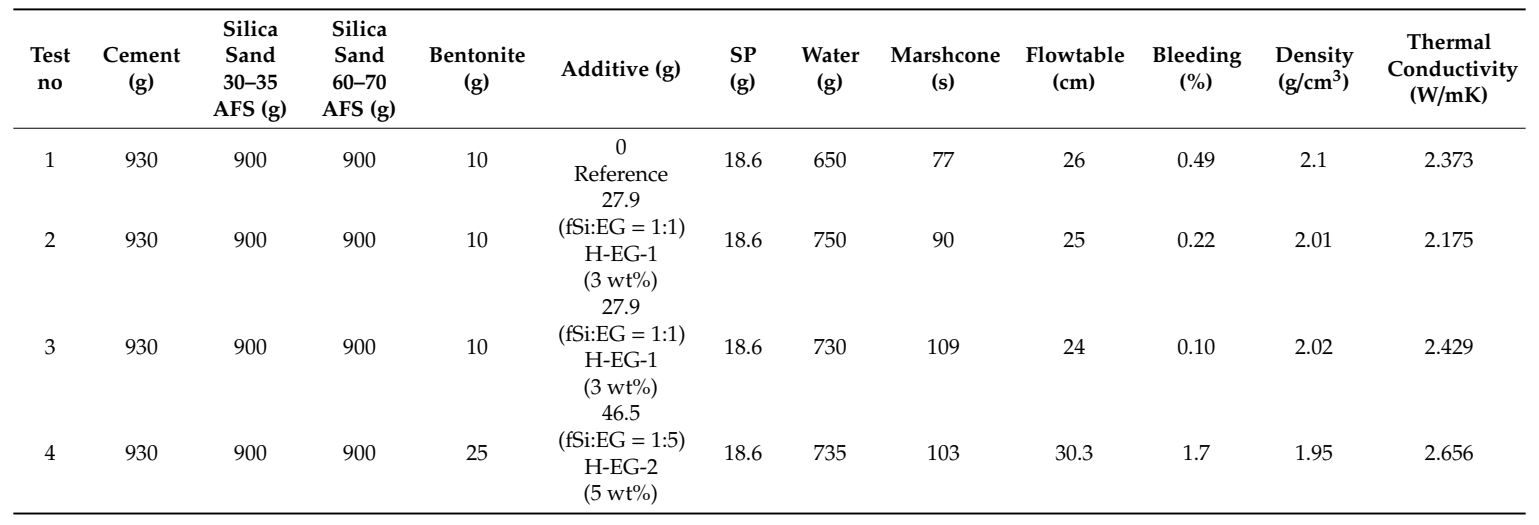

\section{Conclusions}

In the present study, the enhancement of the thermal conductivity of cement-based grouts used in shallow geothermal energy systems was carried out by the development of surface chemistry-controlled expanded graphite (EG)-based hybrid additives. For the functionalization process, APTES as a silane coupling agent with amino functional groups was attached to the silica surface in order to make suitable bridges with the EG surface. Then, a hybridization study was carried out to develop two kinds of EG-based additives by controlling the fSi ratio in order to monitor the influence of the carbon content on the formulated grout mixtures and facilitate their dispersion in aqueous media. XPS characterization confirmed the changes in the $\mathrm{Si}$ and $\mathrm{C}$ ratios on the surface of hybrid structures. The $\mathrm{C}$ atomic percentages of H-EG-1 and H-EG-2 were measured as 50.5 and 88.2, respectively. In addition, the crystallinity degree of the EG-based hybrids was decreased by the addition of amorphous silica. 
Afterwards, the incorporation of the silica/EG hybrid additives in the grout mix with an optimized water demand enhanced the thermal conductivity noticeably compared to that in the reference grout. The result of the thermal conductivity measurement of the grout mix developed with addition of $5 \mathrm{wt} \%$ silica/EG hybrid additive (H-EG-2) showed the highest thermal conductivity value of $2.656 \mathrm{~W} / \mathrm{mK}$. The effect of the water content was also assessed, highlighting the strong influence on the thermal conductivity of the grout composites. In other words, by changing the fSi/EG ratio in the hybrid additive structure from 1:1 to 1:5, the water demand decreased to such an extent that by increasing the additive loading in the grout mix from $3 \%$ to $5 \%$, the water demand was increased only by less than $1 \%$. Consequently, the thermal conductivity showed a noticeable increase of approximately $12 \%$. The increase in the thermal conductivity was attributed to the increase in the EG content in the hybrid additive structure as well as the increase in the additive loading in the grout composition but without a considerable increase in the water demand. Consequently, the modification of EG provided carries significant importance for the performance enhancement of bentonite-based grout mixtures, and functionalization improves the interfacial interactions between grout constituents, thus resulting in the fabrication of high-performing cement composites.

Author Contributions: I.B. and B.S.O. contributed to the preparation of manuscript and gathered all data from the other partners and put them into one structure. I.B. conducted the synthesis experiments and characterization study. In addition, B.S.O. and Y.M. contributed to the experimental design of hybrid structures chemically and the interpretation of their synthesis results. A.C., A.N.G. and P.F. contributed to the grout formulation and sample preparation for thermal conductivity measurements and their analysis and interpretation of data. All authors have read and agreed to the published version of the manuscript.

Funding: This project is supported by Horizon 2020 project of GEOCOND. The grant number is 727583.

Conflicts of Interest: The authors declare no conflict of interest.

\section{References}

1. Sarbu, I.; Sebarchievici, C. Using Ground-Source Heat Pump Systems for Heating/Cooling of Buildings. In Advances in Geothermal Energy; Basel, I.I., Ed.; Rijeka, Croatia: Croatia, 2016.

2. Yang, H.; Cui, P.; Fang, Z. Vertical-borehole ground-coupled heat pumps: A review of models and systems. Appl. Energy 2010, 87, 16-27. [CrossRef]

3. Javadi, H.; Ajarostaghi, S.S.M.; Rosen, M.A.; Pourfallah, M. A comprehensive review of backfill materials and their effects on ground heat exchanger performance. Sustainability 2018, 10, 4486. [CrossRef]

4. Zhang, W.; Yang, H.; Lu, L.; Fang, Z. Investigation on influential factors of engineering design of geothermal heat exchangers. Appl. Therm. Eng. 2015, 84, 310-319. [CrossRef]

5. Lee, H.; Park, S.; Park, S.; Chung, W. Enhanced Detection Systems of Filling Rates Using Carbon Nanotube Cement Grout. Nanomaterials 2019, 10, 10. [CrossRef]

6. Zhang, Q.; Li, H. Experimental investigation on the ice/snow melting performance of CNFP \& MWCNT/cement-based deicing system. In Proceedings of the 6th International Workshop on Advanced Smart Materials and Smart Structures Technology, Dalian, China, 25-26 July 2011.

7. Song, Y.S.; Youn, J. Influence of dispersion states of carbon nanotubes on physical properties of epoxy nanocomposites. Carbon 2005, 43, 1378-1385. [CrossRef]

8. Ajayan, P.M.; Schadler, L.S.; Giannaris, C.; Rubio, A. Single-Walled Carbon Nanotube-Polymer Composites: Strength and Weakness. Adv. Mater. 2000, 12, 750-753. [CrossRef]

9. Delaleux, F.; Py, X.; Olives, R.; Dominguez, A. Enhancement of geothermal borehole heat exchangers performances by improvement of bentonite grouts conductivity. Appl. Therm. Eng. 2012, 33-34, 92-99. [CrossRef]

10. Lee, C.; Lee, K.; Choi, H.; Choi, H.P. Characteristics of thermally-enhanced bentonite grouts for geothermal heat exchanger in South Korea. Sci. China Technol. Sci. 2010, 53, 123-128. [CrossRef]

11. Jobmann, M.; Buntebarth, G. Influence of graphite and quartz addition on the thermo physical properties of bentonite for sealing heat-generating radioactive waste. Appl. Clay Sci. 2009, 44, 206-210. [CrossRef]

12. Peyvandi, A.; Soroushian, P.; Abdol, N.; Balachandra, A.M. Surface-modified graphite nanomaterials for improved reinforcement efficiency in cementitious paste. Carbon 2013, 63, 175-186. [CrossRef] 
13. Karaipekli, A.; Biçer, A.; Sarı, A.; Veer, V. Thermal characteristics of expanded perlite/paraffin composite phase change material with enhanced thermal conductivity using carbon nanotubes. Energy Convers. Manag. 2017, 134, 373-381. [CrossRef]

14. Zhang, Z.; Fang, X. Study on paraffin/expanded graphite composite phase change thermal energy storage material. Energy Convers. Manag. 2006, 47, 303-310. [CrossRef]

15. Bao, X.; Memon, S.A.; Yang, H.; Dong, Z.; Cui, H. Thermal properties of cement-based composites for geothermal energy applications. Materials 2017, 10, 462. [CrossRef] [PubMed]

16. Zhang, B.; Tian, Y.; Jin, X.; Lo, T.; Cui, H. Thermal and Mechanical Properties of Expanded Graphite/Paraffin Gypsum-Based Composite Material Reinforced by Carbon Fiber. Materials 2018, 11, 2205. [CrossRef]

17. Luping, T.; Liu, J.; Wang, N.; Ye, L. Pre-Study of Graphene-Enhanced Cementitious Materials. Available online: https://pdfs.semanticscholar.org/0728/d6fb4819e59eef0c19653ab6785f4d851cfa.pdf (accessed on 5 May 2020).

18. Wang, Q.; Li, S.Y.; Pan, S.; Guo, Z.W. Synthesis and properties of a silane and copolymer-modified graphene oxide for use as a water-reducing agent in cement pastes. New Carbon Mater. 2018, 33, 131-139. [CrossRef]

19. Sharma, N.; Nasimul, S.; Chandra, B.; Yadav, S.; Biswas, K. Diamond \& Related Materials Silica-graphene nanoplatelets and silica-MWCNT composites: Microstructure and mechanical properties. Diam. Relat. Mater. 2018, 87, 186-201.

20. Aly, M.; Hashmi, M.S.J.; Olabi, A.G.; Messeiry, M.; Hussain, A.I. Effect of nano clay particles on mechanical, thermal and physical behaviours of waste-glass cement mortars. Mater. Sci. Eng. A 2011, 528, 7991-7998. [CrossRef]

21. Aly, M.; Hashmi, M.S.J.; Olabi, A.G.; Messeiry, M.; Abadir, E.F.; Hussain, A.I. Effect of colloidal nano-silica on the mechanical and physical behaviour of waste-glass cement mortar. Mater. Des. 2012, 33, 127-135. [CrossRef]

22. Gopalakrishnan, K.; Birgisson, B.; Taylor, P.; Attoh Okine, N. Nanotechnology in Civil Infrastructure; Springer: Berlin/Heidelberg, Germany, 2011; ISBN 978-3-642-16656-3.

23. Shen, C.; Wang, H.; Zhang, T.; Zeng, Y. Silica Coating onto Graphene for Improving Thermal Conductivity and Electrical Insulation of Graphene/polydimethylsiloxane Nanocomposites. J. Mater. Sci. Technol. 2018, 35, 36-43. [CrossRef]

24. Liu, K.; Shang, Y.; Yang, L.; Du, A. Silica/GO Hybrids Reinforced NR: Better Interface Interaction and Dynamic Behavior. J. Elastomers Plast. Available online: https://journals.sagepub.com/doi/10.1177/0095244319877671 (accessed on 12 May 2020).

25. Sadiq, M.M. Reinforcement of Cement-Based Matrices with Graphite a Dissertation. (Civil EngineeringDoctor of Philosophy). Ph.D. Thesis, Michigan State University, Lansing, MI, USA, 2013.

26. Cao, L.; Sinha, T.K.; Tao, L.; Li, H.; Zong, C.; Kim, J.K. Synergistic reinforcement of silanized silica-graphene oxide hybrid in natural rubber for tire-tread fabrication: A latex based facile approach. Compos. Part B Eng. 2019, 161, 667-676. [CrossRef]

27. Shang, Y.; Zhang, D.; Yang, C.; Liu, Y.; Liu, Y. Effect of graphene oxide on the rheological properties of cement pastes. Constr. Build. Mater. 2015, 96, 20-28. [CrossRef]

28. Berktas, I.; Ghafar, A.N.; Fontana, P.; Caputcu, A.; Menceloglu, Y.; Okan, B.S. Facile Synthesis of Graphene from Waste Tire/Silica Hybrid Additives and Optimization Study for the Fabrication of Thermally Enhanced Cement Grouts. Molecules 2020, 25, 886. [CrossRef] [PubMed]

29. Mondal, T.; Bhowmick, A.K.; Krishnamoorti, R. Conducting instant adhesives by grafting of silane polymer onto expanded graphite. ACS Appl. Mater. Interfaces 2014, 6, 16097-16105. [CrossRef]

30. Jiao, X.; Zhang, L.; Qiu, Y.; Yuan, Y. A new adsorbent of $\mathrm{Pb}$ (II) ions from aqueous solution synthesized by mechanochemical preparation of sulfonated expanded graphite. RSC Adv. 2017, 7, 38350-38359. [CrossRef]

31. Desai, S.; Njuguna, J. Enhancement of thermal conductivity of materials using different forms of natural graphite. IOP Conf. Ser. Mater. Sci. Eng. 2012, 40, 012017. [CrossRef]

32. Mengal, N.; Arbab, A.A.; Memon, A.A.; Sahito, I.A.; Jeong, S.H. A promising hybrid graphite counter electrode doped with fumed silica nano-spacers for efficient quasi-solid state dye sensitized solar cells. Electrochim. Acta 2018, 261, 246-255. [CrossRef]

33. Rao, K.S.; Senthilnathan, J.; Liu, Y.-F.; Yoshimura, M. Role of Peroxide Ions in Formation of Graphene Nanosheets by Electrochemical Exfoliation of Graphite. Sci. Rep. 2014, 4, 1-6. [CrossRef] 
34. Shanmugharaj, A.; Kumar, K.T.; Sundari, G.S.; Kumar, E.S.; Ashwini, A.; Ramya, M.; Varsha, P.; Kalaivani, R.; Raghu, S.; Ryu, S. Study on the effect of silica-graphite filler on the rheometric, mechanical, and abrasion loss properties of styrene-butadiene rubber vulcanizates. J. Elastomers Plast. 2019, 51, 359-378. [CrossRef]

35. Ederer, J.; Janoš, P.; Ecorchard, P.; Tolasz, J.; Štengl, V.; Beneš, H.; Perchacz, M.; Pop-Georgievski, O. Determination of amino groups on functionalized graphene oxide for polyurethane nanomaterials: XPS quantitation vs. functional speciation. RSC Adv. 2017, 7, 12464-12473. [CrossRef]

36. Vinoda, B.; Vinuth, M.; Bodke, Y.; Manjanna, J. Photocatalytic Degradation of Toxic Methyl Red Dye Using Silica Nanoparticles Synthesized from Rice Husk Ash. J. Environ. Anal. Toxicol. 2015, 5, 2161-0525.

37. Ferrari, A.C.; Meyer, J.C.; Scardaci, V.; Casiraghi, C.; Lazzeri, M.; Mauri, F.; Piscanec, S.; Jiang, D.; Novoselov, K.S.; Roth, S.; et al. Raman spectrum of graphene and graphene layers. Phys. Rev. Lett. 2006, 97, 187401. [CrossRef]

38. Pavoski, G.; Maraschin, T.; Fim, F.D.C.; Balzaretti, N.M.; Galland, G.B.; Moura, C.S.; Basso, N.R.D.S. Few layer reduced graphene oxide: Evaluation of the best experimental conditions for easy production. Mater. Res. 2017, 20, 53-61. [CrossRef]

(C) 2020 by the authors. Licensee MDPI, Basel, Switzerland. This article is an open access article distributed under the terms and conditions of the Creative Commons Attribution (CC BY) license (http://creativecommons.org/licenses/by/4.0/). 